\title{
Pacific
}

Journal of

Mathematics

\section{PERTURBATIONS OF A CRITICAL FRACTIONAL EQUATION}

Eduardo Colorado, Arturo de Pablo and Urko Sánchez 


\title{
PERTURBATIONS OF A CRITICAL FRACTIONAL EQUATION
}

\author{
Eduardo Colorado, Arturo de Pablo and Urko Sánchez
}

We deal with the following fractional critical problem:

$$
\begin{cases}(-\Delta)^{\alpha / 2} u=|u|^{2 \alpha /(N-\alpha)} u+f(x) & \text { in } \Omega, \\ u=0 & \text { on } \partial \Omega,\end{cases}
$$

where $\Omega \subset \mathbb{R}^{N}$ is a regular bounded domain, $0<\alpha<2$ and $N>\alpha$. Under appropriate conditions on the size of $f$, we prove existence and multiplicity of solutions.

\section{Introduction}

It is well known, using the Pohozhaev identity [1970], that the critical problem

$$
\begin{cases}-\Delta u=|u|^{4 /(N-2)} u & \text { in } \Omega \\ u=0 & \text { on } \partial \Omega\end{cases}
$$

has no positive solution whenever $\Omega$ is a star-shaped domain. Starting from this nonexistence result, in the last decades several perturbations of this problem have been investigated in order to obtain a solution and understand the criticality of the problem. A pioneering work in that sense is the one performed by Brézis and Nirenberg [1983], in which the authors study the existence of positive solutions of the problem

$$
\begin{cases}-\Delta u=|u|^{4 /(N-2)} u+f(x, u) & \text { in } \Omega, \\ u=0 & \text { on } \partial \Omega\end{cases}
$$

where $f(x, u)=f(u)=\lambda u$, with $\lambda \in \mathbb{R}$ and $N>2$. Among other extensions, we highlight the work [Ambrosetti et al. 1994], where the authors studied the case $f(u)=\lambda|u|^{q-2} u$ with $1<q<2$, as well as [Tarantello 1992], in which the case $f(x, u)=f(x)$ was investigated; see also [Rey 1992].

Our purpose here is to study the similar situation that occurs for the fractional Laplacian in a bounded domain and the corresponding critical power.

E. Colorado is partially supported by Spanish Research Projects Ref. MTM2009-10878 and MTM2010-18128. A. de Pablo is partially supported by Spanish Research Project Ref. MTM201125287.

MSC2010: 35A15, 49J35, 35R11.

Keywords: semilinear elliptic equations, fractional Laplacian, critical problem. 
We define the fractional Laplacian in a bounded domain $\Omega$ via its spectral decomposition, namely

$$
(-\Delta)^{\alpha / 2} u=\sum a_{j} \rho_{j}^{\alpha / 2} \varphi_{j},
$$

where $\left\{\rho_{j}, \varphi_{j}\right\}$ is the spectral decomposition of the operator $-\Delta$ in $\Omega$ under zero Dirichlet boundary conditions and the $a_{j}$ are the coefficients of $u$ for the base $\left\{\varphi_{j}\right\}$ in $L^{2}(\Omega)$. A more precise notation would be $(-\Delta)_{\Omega}^{\alpha / 2}$, because the operator strongly depends on the domain $\Omega$, but we omit the subscript since the domain is fixed throughout the paper. We also recall that in the case where the domain under consideration is the whole space $\mathbb{R}^{N}$, the associated fractional Laplacian operator $(-\Delta)_{\mathscr{F}}^{\alpha / 2}$ is defined via Fourier transformation for functions in the Schwartz class:

$$
\left[(-\Delta)_{\mathscr{F}}^{\alpha / 2} g\right]^{\wedge}(\xi)=|\xi|^{\alpha} \hat{g}(\xi)
$$

which gives a different operator.

The critical problem corresponding to (1-2) with the fractional Laplacian is

$$
\begin{cases}(-\Delta)^{\alpha / 2} u=|u|^{2 \alpha /(N-\alpha)} u+f(x, u) & \text { in } \Omega, \\ u=0 & \text { on } \partial \Omega .\end{cases}
$$

First, there is again a (fractional) Pohozhaev-type identity, which in the case $f \equiv 0$ yields, as for the classical problem (1-1), the nonexistence of positive solutions whenever $\Omega$ is a star-shaped domain; see [Brändle et al. 2013]. Note that

$$
\frac{2 \alpha}{N-\alpha}=2_{\alpha}^{*}-2, \quad \text { where } \quad 2_{\alpha}^{*}:=\frac{2 N}{N-\alpha}
$$

is the critical Sobolev exponent associated to $\alpha$.

Next, in the case $f(x, u)=f(u)$, we point out [Barrios et al. 2012], in which an existence and multiplicity result was proved for positive solutions when

$$
f(u)=\lambda|u|^{q-2} u, \quad \lambda>0, \quad 0<\alpha<2, \quad N>\alpha, \quad \text { and } \quad 1<q<\frac{2 N}{N-\alpha} .
$$

The case $\alpha=1$ and $q=2$ was studied previously in [Tan 2011].

In this paper we investigate zero order perturbations, $f(x, u)=f(x)$ small in (1-3), of the critical problem $f \equiv 0$, in relation to the results of [Tarantello 1992] for the classical Laplace operator. Thus, we consider the following problem:

$$
\begin{cases}(-\Delta)^{\alpha / 2} u=|u|^{p-2} u+f(x) & \text { in } \Omega, \\ u=0 & \text { on } \partial \Omega,\end{cases}
$$

where $0<\alpha<2, N>\alpha, p=\frac{2 N}{N-\alpha}$ and $f$ belongs to a suitable space. 
In order to establish the functional setting, we define the function space $H_{0}^{\alpha / 2}(\Omega)$ as the completion of $\mathscr{C}_{0}^{\infty}(\Omega)$ endowed with the norm

$$
\|u\|_{H_{0}^{\alpha / 2}}=\left\|(-\Delta)^{\alpha / 4} u\right\|_{2}=\left(\sum a_{j}^{2} \rho_{j}^{\alpha / 2}\right)^{1 / 2} .
$$

The operator $L(u)=(-\Delta)^{\alpha / 2} u-|u|^{p-2} u$ is well defined from $H_{0}^{\alpha / 2}(\Omega)$ into its dual $H^{-\alpha / 2}(\Omega)$ by the Sobolev inequality; see (2-3) below. Thus it is natural to consider data $f$ in that space: we have $f \in H^{-\alpha / 2}(\Omega)$ if and only if $f=(-\Delta)^{\alpha / 2} g$ with $g \in H_{0}^{\alpha / 2}(\Omega)$; the associated norm is given by $\|f\|_{H^{-\alpha / 2}}=\|g\|_{H_{0}^{\alpha / 2}}$.

Throughout, we will consider solutions of the problem $(\mathrm{P})$ in the following sense:

Definition 1.1. Let $f \in H^{-\alpha / 2}(\Omega)$. We say that $u \in H_{0}^{\alpha / 2}(\Omega)$ is an energy solution to the problem $(\mathrm{P})$ if

$$
\int_{\Omega}(-\Delta)^{\alpha / 4} u(x)(-\Delta)^{\alpha / 4} \psi(x) d x=\int_{\Omega}\left(|u(x)|^{p-2} u(x)+f(x)\right) \psi(x) d x
$$

for every $\psi \in H_{0}^{\alpha / 2}(\Omega)$.

In the sequel we use the simplified notation $\int f=\int f(x) d x$ when no confusion can arise.

The paper is organized as follows: In Section 2 we state the main existence results and establish some preliminaries, and Sections 3 and 4 contain the proofs.

\section{Main results and preliminaries}

We will focus on functions $f \in H^{-\alpha / 2}(\Omega)$ that are small in the following sense:

$$
\int_{\Omega} f \varphi<c(\alpha, N)\|\varphi\|_{H_{0}^{\alpha / 2}}^{(N+\alpha) / \alpha} \text { for all } \varphi \in H_{0}^{\alpha / 2}(\Omega) \text { with }\|\varphi\|_{p}=1,
$$

where

$$
c(\alpha, N)=\frac{2 \alpha}{N-\alpha}\left(\frac{N-\alpha}{N+\alpha}\right)^{\frac{N+\alpha}{2 \alpha}} .
$$

The main result of the paper is the following:

Theorem 2.1. Assume $f \not \equiv 0$ satisfies (2-1). Then the problem $(\mathrm{P})$ has at least two solutions. Moreover, if $f \geq 0$ a.e. in $\Omega$, then these solutions are nonnegative a.e. in $\Omega$.

We will also prove that, if we relax the strict inequality in condition (2-1) by replacing it with the condition

$$
\int_{\Omega} f \varphi \leq c(\alpha, N)\|\varphi\|_{H_{0}^{\alpha / 2}}^{(N+\alpha) / \alpha} \text { for all } \varphi \in H_{0}^{\alpha / 2}(\Omega) \text { with }\|\varphi\|_{p}=1,
$$

then we still obtain the existence of at least one solution: 
Theorem 2.2. Assume $f \not \equiv 0$ satisfies (2-2). Then the problem $(\mathrm{P})$ has at least one solution. Moreover, if $f$ is nonnegative a.e. in $\Omega$ then this solution is nonnegative a.e. in $\Omega$.

For the fractional Laplacian defined above and $N>\alpha$, the following Sobolev inequality holds:

$$
\int_{\Omega}\left|(-\Delta)^{\alpha / 4} \varphi\right|^{2} \geq S(\alpha, N)\left(\int_{\Omega}|\varphi|^{2 N /(N-\alpha)}\right)^{\frac{N-\alpha}{N}} \quad \text { for all } \varphi \in H_{0}^{\alpha / 2}(\Omega) .
$$

See, for example, [Brändle et al. 2013], where the inequality is proved as a consequence of the Hardy-Littlewood-Sobolev inequality [Hardy and Littlewood 1928; Sobolev 1938]. In the case of $\mathbb{R}^{N}$ and $(-\Delta)_{\mathscr{F}}^{\alpha / 4}$, it takes the form

$$
\int_{\mathbb{R}^{N}}\left|(-\Delta)_{\mathscr{F}}^{\alpha / 4} \varphi\right|^{2} \geq S(\alpha, N)\left(\int_{\mathbb{R}^{N}}|\varphi|^{2 N /(N-\alpha)}\right)^{\frac{N-\alpha}{N}} \quad \text { for all } \varphi \in \mathscr{Y}\left(\mathbb{R}^{N}\right) \text {. }
$$

The value of $S(\alpha, N)$ can be seen, for instance, in [Lieb 1983]. It is independent of the domain and is not attained in any bounded domain, although it is attained in $\mathbb{R}^{N}$.

The condition (2-1) is equivalent to

$$
\int_{\Omega} f \varphi<c(\alpha, N) \frac{\|\varphi\|_{H_{0}^{\alpha / 2}}^{(N+\alpha) / \alpha}}{\|\varphi\|_{p}^{N / \alpha}} \text { for all } \varphi \in H_{0}^{\alpha / 2}(\Omega) \backslash\{0\} .
$$

Moreover, since

$$
\int_{\Omega} f \varphi \leq\|f\|_{H^{-\alpha / 2}}\|\varphi\|_{H_{0}^{\alpha / 2}}
$$

using the Sobolev inequality (2-3) we obtain the following sufficient condition for $f$ to satisfy (2-1):

$$
\|f\|_{H^{-\alpha / 2}} \leq c(\alpha, N) S(\alpha, N)^{N / 2 \alpha} .
$$

Remarks. (1) An assumption on the size of $f$ like (2-1) is necessary in order to find solutions of problem (P). For example, if $f$ is a sufficiently large positive constant, then problem $(\mathrm{P})$ has no solutions.

(2) Condition (2-7) seems not to be sharp, in view of the result in [Castro and Zuluaga 1993] for the case $\alpha=2$, which could also be proved in our functional framework.

The associated energy functional to problem $(\mathrm{P})$ is given by

$$
I(u)=\frac{1}{2} \int_{\Omega}\left|(-\Delta)^{\alpha / 4} u\right|^{2}-\frac{1}{p} \int_{\Omega}|u|^{p}-\int_{\Omega} f u .
$$


Clearly, critical points of $I$ correspond to solutions of (P) in the sense of (1-4). Indeed, one of the solutions we will construct in the proof of Theorem 2.1 is a local minimum of $I$ in $H_{0}^{\alpha / 2}(\Omega)$.

\section{Proof of Theorem 2.1}

First solution. We start with the definition of the Nehari manifold associated to problem $(\mathrm{P})$ :

$$
\mathcal{N}=\left\{u \in H_{0}^{\alpha / 2}(\Omega): u \neq 0,\left\langle I^{\prime}(u), u\right\rangle=0\right\} .
$$

It is natural to look for solutions in this manifold. Note that the condition $u \in \mathcal{N}$ is equivalent to the identity

$$
\|u\|_{H_{0}^{\alpha / 2}}^{2}=\|u\|_{p}^{p}+\int_{\Omega} f u .
$$

Therefore the functional $I$ restricted to $\mathcal{N}$ takes the equivalent forms

$$
I(u)=\frac{\alpha}{2 N}\|u\|_{H_{0}^{\alpha / 2}}^{2}-\frac{N+\alpha}{2 N} \int_{\Omega} f u=\frac{\alpha}{2 N}\|u\|_{p}^{p}-\frac{1}{2} \int_{\Omega} f u .
$$

We will use both expressions in the sequel. In particular, using the first one we deduce that the functional $I$ is bounded from below on $\mathcal{N}$ :

$$
I(u) \geq \frac{\alpha}{2 N}\|u\|_{H_{0}^{\alpha / 2}}^{2}-\frac{N+\alpha}{2 N}\|f\|_{H^{-\alpha / 2}}\|u\|_{H_{0}^{\alpha / 2}} \geq-\frac{(N+\alpha)^{2}}{8 N \alpha}\|f\|_{H^{-\alpha / 2}}^{2},
$$

where the last step is a consequence of the minimization of the function

$$
\alpha t^{2}-(N+\alpha)\|f\|_{H^{-\alpha / 2}} t .
$$

Remark. Taking (3-3) into account, it makes sense to define

$$
c_{0}=\inf _{\mathcal{N}} I>-\infty,
$$

although the functional is not bounded from below in the whole space $H_{0}^{\alpha / 2}(\Omega)$.

Note that if $u_{0}$ is a local minimum of $I$ in $H_{0}^{\alpha / 2}(\Omega)$, then necessarily

$$
\left\|u_{0}\right\|_{H_{0}^{\alpha / 2}}^{2}-(p-1)\left\|u_{0}\right\|_{p}^{p} \geq 0 .
$$

In fact, as we will prove in Lemma 3.4, this inequality is strict; namely,

$$
\left\|u_{0}\right\|_{H_{0}^{\alpha / 2}}^{2}-(p-1)\left\|u_{0}\right\|_{p}^{p}>0 .
$$

In the same way, if $u_{0}$ is a local maximum of $I$, we have

$$
\left\|u_{0}\right\|_{H_{0}^{\alpha / 2}}^{2}-(p-1)\left\|u_{0}\right\|_{p}^{p}<0 .
$$


Thus, we first minimize the restriction of the functional $I$ to $\mathcal{N}$ in order to find a critical point and therefore a solution to the problem $(\mathrm{P})$. As we will see, $c_{0}$ is achieved. To prove that, we start with some preliminary results.

Lemma 3.1. Let $f \not \equiv 0$ satisfy (2-1). Given $u \in H_{0}^{\alpha / 2}(\Omega)$, assume $\int_{\Omega} f u>0$. Then there exist unique numbers $\sigma=\sigma(u)>0$ and $\tau=\tau(u)>\sigma$ with $\sigma u, \tau u \in \mathcal{N}$ and such that (3-5) is satisfied with $u_{0}=\sigma u$ and (3-6) with $u_{0}=\tau u$.

Proof. Let $\theta(t)=t\|u\|_{H_{0}^{\alpha / 2}}^{2}-t^{p-1}\|u\|_{p}^{p}$. The maximum value of this function occurs at

$$
t_{M}=\left(\frac{(N-\alpha)\|u\|_{H_{0}^{\alpha / 2}}^{2}}{(N+\alpha)\|u\|_{p}^{p}}\right)^{\frac{N-\alpha}{2 \alpha}},
$$

and

$$
\theta\left(t_{M}\right)=\frac{2 \alpha}{N-\alpha}\left(\frac{N-\alpha}{N+\alpha}\right)^{\frac{N+\alpha}{2 \alpha}} \frac{\|u\|_{H_{0}^{\alpha / 2}}^{(N+\alpha) / \alpha}}{\|u\|_{p}^{N / \alpha}}=c(\alpha, N) \frac{\|u\|_{H_{0}^{\alpha / 2}}^{(N+\alpha) / \alpha}}{\|u\|_{p}^{N / \alpha}} .
$$

Note that $\theta$ is a concave function, increasing on $\left(0, t_{M}\right)$ and decreasing on $\left(t_{M}, \infty\right)$, with $\lim _{t \rightarrow \infty} \theta(t)=-\infty$. By (2-5) we get $0<\int_{\Omega} f u<\theta\left(t_{M}\right)$. Thus there exist two unique values $0<\sigma<t_{M}<\tau$ such that

$$
\theta(\tau)=\int_{\Omega} f u=\theta(\sigma), \quad \theta^{\prime}(\tau)<0<\theta^{\prime}(\sigma) .
$$

Multiplying in the previous expression by $\tau$, we have

$$
0=\tau \theta(\tau)-\tau \int_{\Omega} f u=\|\tau u\|_{H_{0}^{\alpha / 2}}^{2}-\|\tau u\|_{p}^{p}-\int_{\Omega} \tau f u
$$

thus $\tau u \in \mathcal{N}$. Moreover,

$$
\|\tau u\|_{H_{0}^{\alpha / 2}}^{2}-(p-1)\|\tau u\|_{p}^{p}=\tau^{2} \theta^{\prime}(\tau)<0 .
$$

Arguing in a similar way for $\sigma$, we obtain $\sigma u \in \mathcal{N}$ and

$$
\|\sigma u\|_{H_{0}^{\alpha / 2}}^{2}-(p-1)\|\sigma u\|_{p}^{p}=\sigma^{2} \theta^{\prime}(\sigma)>0 .
$$

Observe that without the condition $\int_{\Omega} f u>0$ we still can find a value $\tau>0$ with $\tau u \in \mathcal{N}$ satisfying (3-5). Conversely, the condition $\int_{\Omega} f u>0$ is guaranteed for any function $u \in \mathcal{N}$ that satisfies (3-5).

We notice that the purpose of the strict condition (2-1) on $f$ is just to obtain $\int_{\Omega} f u<\theta\left(t_{M}\right)$. It also appears to be of importance in Lemma 3.3 below. It is known that, when one deals with the problem associated to the standard Laplacian, and under certain hypotheses, the condition (2-1) is not sharp; see [Castro and Zuluaga 1993]. We suspect that a similar fact can occur in our case. 
Corollary 3.2. Under the hypotheses of Lemma 3.1, we have

$$
I(\tau u)=\max _{t \geq \sigma} I(t u) \text { and } I(\sigma u)=\min _{0 \leq t \leq \tau} I(t u) .
$$

Proof. It is straightforward once we notice that the function $g(t)=I(t u)$ satisfies $g^{\prime}(t)=\theta(t)-\int_{\Omega} f u$.

The next property uses a technical result analogous to Lemma 2.2 in [Tarantello 1992]. The proof follows almost word by word the one in that paper; see also [Brézis and Nirenberg 1989]. We only have to adapt the calculations to the functional framework of the fractional Laplacian. We leave the details to the interested reader.

Lemma 3.3. Let $f \not \equiv 0$ satisfy (2-1). Then

$$
\mu_{0}:=\inf _{\substack{u \in H_{0}^{\alpha / 2}(\Omega) \\\|u\|_{p}=1}}\left(c(\alpha, N)\|u\|_{H_{0}^{\alpha / 2}}^{(N+\alpha) / \alpha}-\int_{\Omega} f u\right)
$$

is achieved, and moreover $\mu_{0}>0$.

The proof of this lemma is a straightforward adaptation to our setting of the similar one in the classical case; see Lemma 2.2 in [Tarantello 1992], which is inspired by the corresponding result in [Brézis and Nirenberg 1989].

The following lemma establishes a crucial property for minima of the functional; see inequality (3-5).

Lemma 3.4. Let $f \not \equiv 0$ satisfy (2-1) and let $u \in \mathcal{N}$. Then

$$
\|u\|_{H_{0}^{\alpha / 2}}^{2}-(p-1)\|u\|_{p}^{p} \neq 0 .
$$

Proof. Consider the functional, defined for $u \in H_{0}^{\alpha / 2}(\Omega), u \neq \equiv$, by

$$
\phi(u)=c(\alpha, N) \frac{\|u\|_{H_{0}^{\alpha / 2}}^{(N+\alpha) / \alpha}}{\|u\|_{p}^{N / \alpha}}-\int_{\Omega} f u .
$$

If $\|u\|_{p}=1$, we have

$$
\phi(t u)=t\left(c(\alpha, N)\|u\|_{H_{0}^{\alpha / 2}}^{(N+\alpha) / \alpha}-\int_{\Omega} f u\right) .
$$

Thus, given $\gamma>0$ (to be chosen later), by Lemma 3.3 we have

$$
\inf _{\|u\|_{p} \geq \gamma} \phi(u) \geq \gamma \mu_{0} .
$$

Note that this infimum is also positive.

Now we suppose, for a contradiction, that there exists $u \in \mathcal{N}$ such that

$$
\|u\|_{H_{0}^{\alpha / 2}}^{2}-(p-1)\|u\|_{p}^{p}=0 .
$$


By the Sobolev inequality (2-3), we obtain

$$
S(\alpha, N)\|u\|_{p}^{2}-(p-1)\|u\|_{p}^{p} \leq 0,
$$

which implies

$$
\|u\|_{p} \geq\left(\frac{S(\alpha, N)}{p-1}\right)^{1 /(p-2)}=: \gamma .
$$

Now, substituting (3-10) into (3-1), we get

$$
0=\|u\|_{H_{0}^{\alpha / 2}}^{2}-\|u\|_{p}^{p}-\int_{\Omega} f u=(p-2)\|u\|_{p}^{p}-\int_{\Omega} f u .
$$

Finally, by (3-9) and (3-11), we conclude that

$$
\begin{aligned}
0<\gamma \mu_{0} \leq \phi(u) & =(p-2)\left(\frac{N-\alpha}{N+\alpha}\right)^{\frac{N+\alpha}{2 \alpha}} \frac{\|u\|_{H_{0}^{\alpha / 2}}^{(N+\alpha) / \alpha}}{\|u\|_{p}^{N / \alpha}}-\int_{\Omega} f u \\
& =(p-2)\left[\left(\frac{N-\alpha}{N+\alpha}\right)^{\frac{N+\alpha}{2 \alpha}} \frac{\|u\|_{H_{0}^{\alpha / 2}}^{(N+\alpha) / \alpha}}{\|u\|_{p}^{N / \alpha}}-\|u\|_{p}^{p}\right] \\
& =(p-2)\|u\|_{p}^{p}\left[\left(\frac{(N-\alpha)\|u\|_{H_{0}^{\alpha / 2}}^{2}}{(N+\alpha)\|u\|_{p}^{p}}\right)^{\frac{N-\alpha}{2 \alpha}}-1\right]=0,
\end{aligned}
$$

which is a contradiction.

Lemma 3.5. Let $f \not \equiv 0$ be a function satisfying (2-1). Given $u \in \mathcal{N}$, there exists a positive function $\mu_{u}: H_{0}^{\alpha / 2}(\Omega) \rightarrow \mathbb{R}$, differentiable in a neighborhood $U_{0}$ of the origin in $H_{0}^{\alpha / 2}(\Omega)$, such that

$$
\mu_{u}(0)=1, \quad \mu_{u}(z)(u-z) \in \mathcal{N},
$$

and

$$
\left\langle\mu_{u}^{\prime}(0), z\right\rangle=\frac{2 \int_{\Omega}(-\Delta)^{\alpha / 4} u(-\Delta)^{\alpha / 4} z-p \int_{\Omega}|u|^{p-2} u z-\int_{\Omega} f z}{\|u\|_{H_{0}^{\alpha / 2}}^{2}-(p-1)\|u\|_{p}^{p}} \quad \text { for all } z \in U_{0} .
$$

Proof. Consider the function

$$
F(\mu, z)=\mu\|u-z\|_{H_{0}^{\alpha / 2}(\Omega)}^{2}-\mu^{p-1}\|u-z\|_{p}^{p}-\int_{\Omega} f(u-z) .
$$

By Lemma 3.4, we have

$$
\frac{\partial F}{\partial \mu}(1,0)=\|u\|_{H_{0}^{\alpha / 2}}^{2}-(p-1)\|u\|_{p}^{p} \neq 0 .
$$

We complete the proof by applying the implicit function theorem to the function $F$ at the point $(1,0)$. 
We are now in a position to prove one of the main results of the paper.

Proposition 3.6. The functional I possesses a local minimum in $H_{0}^{\alpha / 2}(\Omega)$. In particular, $(\mathrm{P})$ has a solution. Moreover, if $f$ is nonnegative a.e. in $\Omega$, this solution is nonnegative a.e. in $\Omega$.

Proof. Consider $v$, the unique solution to the equation $(-\Delta)^{\alpha / 2} v=f$ in $H_{0}^{\alpha / 2}(\Omega)$. Let $\sigma=\sigma(v)$ be as defined in Lemma 3.1. Since $\sigma(v) v \in \mathcal{N}$, we have

$$
\begin{aligned}
I(\sigma v) & =\frac{\sigma^{2}}{2}\|v\|_{H_{0}^{\alpha / 2}}^{2}-\frac{\sigma^{p}}{p}\|v\|_{p}^{p}-\sigma\|v\|_{H_{0}^{\alpha / 2}}^{2} \\
& =-\frac{\sigma^{2}}{2}\|v\|_{H_{0}^{\alpha / 2}}^{2}+\frac{N+\alpha}{2 N} \sigma^{p}\|v\|_{p}^{p} \\
& <-\frac{\alpha \sigma^{2}}{2 N}\|v\|_{H_{0}^{\alpha / 2}}^{2}=-\frac{\alpha \sigma^{2}}{2 N}\|f\|_{H^{-\alpha / 2}}^{2} .
\end{aligned}
$$

Then, by (3-3) and (3-13), the infimum in (3-4) satisfies the estimate

$$
-\frac{(N+\alpha)^{2}}{8 N \alpha}\|f\|_{H^{-\alpha / 2}}^{2} \leq c_{0}<-\frac{\alpha \sigma^{2}}{2 N}\|f\|_{H^{-\alpha / 2}}^{2}<0 .
$$

The expression (3-2) shows that the restriction of the functional $I$ to $\mathcal{N}$ is weakly lower semicontinuous. Therefore, by Ekeland's variational principle [1974], we obtain a minimizing sequence of the functional $I$ constrained to $\mathcal{N}$, i.e., $\left\{u_{n}\right\} \subset \mathcal{N}$ such that, for every $n \in \mathbb{N}$,

(i) $I\left(u_{n}\right)<c_{0}+\frac{1}{n}$ and (ii) $\frac{1}{n}\left\|u_{n}-v\right\|_{H_{0}^{\alpha / 2}} \geq I\left(u_{n}\right)-I(v)$ for all $v \in \mathcal{N}$.

Combining (i), (3-14) and (3-2), we have

$$
I\left(u_{n}\right)=\frac{\alpha}{2 N}\left\|u_{n}\right\|_{H_{0}^{\alpha / 2}}^{2}-\frac{N+\alpha}{2 N} \int_{\Omega} f u_{n}<c_{0}+\frac{1}{n}<-\frac{\alpha \sigma^{2}}{2 N}\|f\|_{H^{-\alpha / 2}}^{2}
$$

for $n$ large enough. Therefore

$$
\frac{\alpha \sigma^{2}}{N+\alpha}\|f\|_{H^{-\alpha / 2}}^{2} \leq \int_{\Omega} f u_{n} \quad \text { and } \quad\left\|u_{n}\right\|_{H_{0}^{\alpha / 2}}^{2} \leq \frac{N+\alpha}{\alpha} \int_{\Omega} f u_{n} .
$$

These inequalities, together with (2-6), give

$$
\frac{\alpha \sigma^{2}}{N+\alpha}\|f\|_{H^{-\alpha / 2}} \leq\left\|u_{n}\right\|_{H_{0}^{\alpha / 2}} \leq \frac{N+\alpha}{\alpha}\|f\|_{H^{-\alpha / 2}} .
$$

Thus, we have (for a subsequence) that $u_{n} \rightarrow u_{0}$ weakly in $H^{\alpha / 2}(\Omega)$, with $u_{0} \not \equiv 0$. We claim that $\left\|I^{\prime}\left(u_{0}\right)\right\|_{H^{-\alpha / 2}}=0$. Take $z \in H_{0}^{\alpha / 2}(\Omega)$ with $\|z\|_{H_{0}^{\alpha / 2}}=1$. By Lemma 3.5, for every $n \in \mathbb{N}$, there exists a positive function $\mu_{u_{n}}$ such that

$$
w_{\delta}=\mu_{u_{n}}(\delta z)\left(u_{n}-\delta z\right) \in \mathcal{N}
$$

for $\delta>0$ small enough. Set $t_{n}(\delta)=\mu_{u_{n}}(\delta z)$. Putting $v=w_{\delta}$ in (ii) and using the 
mean value theorem, we have

$$
\frac{1}{n}\left\|w_{\delta}-u_{n}\right\|_{H_{0}^{\alpha / 2}} \geq\left(1-t_{n}(\delta)\right)\left\langle I^{\prime}\left(w_{\delta}\right), u_{n}\right\rangle+\delta t_{n}(\delta)\left\langle I^{\prime}\left(w_{\delta}\right), z\right\rangle+o(\delta) .
$$

Dividing by $\delta$ and taking the limit as $\delta$ goes to 0 , we have

$$
\frac{1}{n}\left(1+\left|t_{n}^{\prime}(0)\right|\left\|u_{n}\right\|_{H_{0}^{\alpha / 2}}\right) \geq\left\|I^{\prime}\left(u_{n}\right)\right\|_{H^{-\alpha / 2}}
$$

with $\left|t_{n}^{\prime}(0)\right|=\left\langle\mu_{u_{n}}^{\prime}(0), z\right\rangle$, so that, by (3-16), we get

$$
\left\|I^{\prime}\left(u_{n}\right)\right\|_{H^{-\alpha / 2}} \leq \frac{1}{n}\left(1+\frac{N+\alpha}{\alpha}\left|t_{n}^{\prime}(0)\right|\|f\|_{H^{-\alpha / 2}}\right) .
$$

Thus we are done once we prove that $\left|t_{n}^{\prime}(0)\right|$ is uniformly bounded. By Lemma 3.5 and (3-16) we obtain

$$
\left|t_{n}^{\prime}(0)\right| \leq \frac{C}{\left|\left\|u_{n}\right\|_{H_{0}^{\alpha / 2}}^{2}-(p-1)\left\|u_{n}\right\|_{p}^{p}\right|}
$$

for some constant $C$. Assume, for a contradiction, that

$$
\left\|u_{n}\right\|_{H_{0}^{\alpha / 2}}^{2}-(p-1)\left\|u_{n}\right\|_{p}^{p} \rightarrow 0 \quad \text { as } n \rightarrow \infty .
$$

From (3-18) and (3-1) we deduce the estimate

$$
\int_{\Omega} f u_{n}=(p-2)\left\|u_{n}\right\|_{p}^{p}+o(1) .
$$

Moreover, from (3-16) we derive that $\left\|u_{n}\right\|_{p} \geq \gamma$ for some constant $\gamma>0$. Thus, reasoning as in Lemma 3.4, we get

$$
\begin{aligned}
0 & <\gamma^{(N+\alpha) / 2} \mu_{0} \leq\left\|u_{n}\right\|_{H_{0}^{\alpha / 2}}^{\alpha / N} \phi\left(u_{n}\right) \\
& =(p-2)\left[\left(\frac{(N-\alpha)\left\|u_{n}\right\|_{H_{0}^{\alpha / 2}}^{2}}{N+\alpha}\right)^{\frac{N-\alpha}{2 \alpha}}-\left(\left\|u_{n}\right\|_{p}^{p}\right)^{(N-\alpha) / 2 \alpha}\right] \rightarrow 0,
\end{aligned}
$$

which leads to a contradiction. Therefore $\left\|I^{\prime}\left(u_{0}\right)\right\|_{H^{-\alpha / 2}}=0$, and we have obtained a weak solution of $(\mathrm{P})$.

To obtain strong convergence, we proceed as usual. Recalling that $I$ is weakly lower semicontinuous in $\mathcal{N}$, we get

$$
c_{0} \leq I\left(u_{0}\right) \leq \lim _{n \rightarrow \infty} I\left(u_{n}\right)=c_{0} .
$$

This implies, using (3-2), the limits

$$
\lim _{n \rightarrow \infty}\left\|u_{n}\right\|_{H_{0}^{\alpha / 2}}=\left\|u_{0}\right\|_{H_{0}^{\alpha / 2}}, \quad \lim _{n \rightarrow \infty}\left\|u_{n}\right\|_{p}=\left\|u_{0}\right\|_{p} .
$$

To see that $u_{0}$ is a local minimum in $H_{0}^{\alpha / 2}(\Omega)$, we first show that (3-5) holds. In fact, since $u_{0} \in \mathcal{N}$ and also $\int_{\Omega} f u_{0}>0$ by (3-15), it is clear that one of the values $\sigma\left(u_{0}\right)$ 
or $\tau\left(u_{0}\right)$ given by Lemma 3.1 equals 1. Assume, for a contradiction (see Lemma 3.4), that $u_{0}$ satisfies (3-6), i.e., $\sigma\left(u_{0}\right)<\tau\left(u_{0}\right)=1$. By Corollary 3.2, $I\left(\sigma\left(u_{0}\right) u_{0}\right)<I\left(u_{0}\right)$, which contradicts the fact that $u_{0}$ is the infimum in $\mathcal{N}$. Hence $u_{0}$ satisfies (3-5) and $\sigma\left(u_{0}\right)=1$. We remark that having the strict inequality in (2-5) is crucial in the present argument. In particular, we have obtained $1=\sigma\left(u_{0}\right)<t_{M}<\tau\left(u_{0}\right)$, or

$$
1<\left(\frac{(N-\alpha)\left\|u_{0}\right\|_{H_{0}^{\alpha / 2}}^{2}}{(N+\alpha)\left\|u_{0}\right\|_{p}^{p}}\right)^{\frac{N-\alpha}{2 \alpha}},
$$

which is the same. Take $\varepsilon>0$ small enough such that

$$
1<\left(\frac{(N-\alpha)\left\|u_{0}-z\right\|_{H_{0}^{\alpha / 2}}^{2}}{(N+\alpha)\left\|u_{0}-z\right\|_{p}^{p}}\right)^{\frac{N-\alpha}{2 \alpha}}=: t_{M, \varepsilon}
$$

for $\|z\|_{H_{0}^{\alpha / 2}}<\varepsilon$. By Lemma 3.5, there exists a positive function $\mu_{u_{0}}: H_{0}^{\alpha / 2}(\Omega) \rightarrow \mathbb{R}$ such that $\mu_{u_{0}}(z)\left(u_{0}-z\right) \in \mathcal{N}$ for every $\|z\|_{H_{0}^{\alpha / 2}}<\varepsilon$, with $\varepsilon$ smaller if necessary. Indeed, by continuity we have $\mu_{u_{0}}(z)<t_{M, \varepsilon}$ for $\varepsilon>0$ sufficiently small. Thus we get that $\mu_{u_{0}}(z)\left(u_{0}-z\right)$ satisfies (3-5), and as a consequence of Lemma 3.1 and Corollary 3.2 applied to $u_{0}-z$, we obtain

$$
I\left(s\left(u_{0}-z\right)\right) \geq I\left(\mu_{u_{0}}(z)\left(u_{0}-z\right)\right) \geq I\left(u_{0}\right) \quad \text { for all } s \in\left(0, t_{M, \varepsilon}\right) .
$$

Since by (3-20) we can take $s=1$, we conclude that $I\left(u_{0}-z\right) \geq I\left(u_{0}\right)$ for every $\|z\|_{H_{0}^{\alpha / 2}}<\varepsilon$, i.e, $u_{0}$ is a local minimum in $H_{0}^{\alpha / 2}(\Omega)$.

To finish, we assume that $f \geq 0$. Then it follows that $\int_{\Omega} f\left|u_{0}\right|>0$. Take $\sigma=\sigma\left(\left|u_{0}\right|\right)>0$ and $\tau=\tau\left(\left|u_{0}\right|\right)>\sigma$. We have

$$
\left\|u_{0}\right\|_{p}^{p}+\int_{\Omega} f u_{0}=\left\|u_{0}\right\|_{H_{0}^{\alpha / 2}}^{2}>(p-1)\left\|u_{0}\right\|_{p}^{p}
$$

and, since $\tau\left|u_{0}\right|$ satisfies (3-6), we get

$$
\tau^{p}\left\|u_{0}\right\|_{p}^{p}+\tau \int_{\Omega} f\left|u_{0}\right|=\tau^{2}\left\|\left|u_{0}\right|\right\|_{H_{0}^{\alpha / 2}}^{2}<(p-1) \tau^{p}\left\|u_{0}\right\|_{p}^{p} .
$$

Thus,

$$
(p-2)\left\|u_{0}\right\|_{p}^{p}<\int_{\Omega} f u_{0} \leq \int_{\Omega} f\left|u_{0}\right| \leq(p-2) \tau^{p-1}\left\|u_{0}\right\|_{p}^{p},
$$

which implies $\tau>1$. Therefore, by Corollary 3.2, we have

$$
I\left(u_{0}\right) \leq I\left(\sigma\left|u_{0}\right|\right) \leq I\left(\left|u_{0}\right|\right) .
$$

On the other hand, by the generalized Stroock-Varopoulos inequality [de Pablo et al. 2012], we have

$$
\int_{\Omega}\left|(-\Delta)^{\alpha / 4}\right| u_{0}||^{2} \leq \int_{\Omega}\left|(-\Delta)^{\alpha / 4} u_{0}\right|^{2},
$$


which implies $I\left(\left|u_{0}\right|\right) \leq I\left(u_{0}\right)$. As a consequence, $I\left(u_{0}\right)=I\left(\left|u_{0}\right|\right), \sigma=1$, and thus $\left|u_{0}\right| \in \mathcal{N}$ is a solution.

Second solution. We will look for the second solution using a classical approach that relies on the well-known mountain pass theorem; see [Ambrosetti and Rabinowitz 1973]. Recall that $\left\{u_{n}\right\} \subset H_{0}^{\alpha / 2}(\Omega)$ is a Palais-Smale (PS for short) sequence of level $c$ for $I$ if $I\left(u_{n}\right) \rightarrow c$ and $\left\|I^{\prime}\left(u_{n}\right)\right\|_{H^{-\alpha / 2}} \rightarrow 0$ as $n \rightarrow \infty$. Moreover, we say that $I$ satisfies a PS condition of level $c$ ( $\mathrm{PS}_{c}$ for short) if every PS sequence of level $c$ for $I$ has a convergent subsequence in $H_{0}^{\alpha / 2}(\Omega)$. As is usual in critical problems, the functional $I$ does not satisfy a global PS condition, i.e., a $\mathrm{PS}_{c}$ condition for every $c$. Our aim is to prove that $I$ satisfies a $\mathrm{PS}_{c}$ condition for $c$ below a precise critical level $c^{*}$. We define

$$
c^{*}=c_{0}+\frac{\alpha}{2 N} S(\alpha, N)^{N / \alpha} .
$$

This value, which is obtained in the next lemma, also appears in several other contexts, for instance when one applies the concentration-compactness principle to critical problems; see [Ambrosetti et al. 1994; Brézis and Nirenberg 1983; Hardy and Littlewood 1928; Lions 1985] for the standard case, and, for example, [Barrios et al. 2012] for the fractional case, and [Barrios et al. 2014; Servadei and Valdinoci $\geq 2014$ ] for different nonlocal operators which include a different fractional Laplacian.

Lemma 3.7. The functional I satisfies a local $P S_{c}$ condition for any $c<c^{*}$.

Proof. Let $\left\{u_{n}\right\} \subset H_{0}^{\alpha / 2}(\Omega)$ be a PS sequence of level $c<c^{*}$. It is easy to check that the $u_{n}$ are uniformly bounded in $H^{\alpha / 2}(\Omega)$. Thus, there exists a subsequence (still denoted by $u_{n}$ ) such that $u_{n} \rightarrow z_{0}$ weakly in $H_{0}^{\alpha / 2}(\Omega)$. As a consequence, $z_{0} \in H_{0}^{\alpha / 2}(\Omega)$ is a solution of $(\mathrm{P})$.

We rewrite $u_{n}$ as $u_{n}=u_{0}+\phi_{n}$ with $\phi_{n} \rightarrow 0$. Applying the Brézis-Lieb lemma [1983] we get

$$
\left\|u_{n}\right\|_{p}^{p}=\left\|u_{0}\right\|_{p}^{p}+\left\|\phi_{n}\right\|_{p}^{p}+o(1) .
$$

On one hand, by (3-22) and taking $n$ large enough we have

$$
\begin{aligned}
c^{*}>I\left(u_{n}\right) & =I\left(u_{0}\right)+\frac{1}{2}\left\|\phi_{n}\right\|_{H_{0}^{\alpha / 2}}^{2}-\frac{1}{p}\left\|\phi_{n}\right\|_{p}^{p}+o(1) \\
& \geq c_{0}+\frac{1}{2}\left\|\phi_{n}\right\|_{H_{0}^{\alpha / 2}}^{2}-\frac{1}{p}\left\|\phi_{n}\right\|_{p}^{p}+o(1) .
\end{aligned}
$$

Hence, by the definition of $c^{*}$ in (3-21), we obtain

$$
\frac{1}{2}\left\|\phi_{n}\right\|_{H_{0}^{\alpha / 2}}^{2}-\frac{1}{p}\left\|\phi_{n}\right\|_{p}^{p}<\frac{\alpha}{2 N} S(\alpha, N)^{N / \alpha}+o(1) .
$$


Taking into account that $\left\{u_{n}\right\}$ is a PS sequence, we have

$$
\begin{aligned}
o(1) & =\left\langle I^{\prime}\left(u_{n}\right), u_{n}\right\rangle=\left\|u_{n}\right\|_{H_{0}^{\alpha / 2}}^{2}-\left\|u_{n}\right\|_{p}^{p}-\int_{\Omega} f u_{n} \\
& =\left\|u_{0}\right\|_{H_{0}^{\alpha / 2}}^{2}-\left\|u_{0}\right\|_{p}^{p}-\int_{\Omega} f u_{0}+\left\|\phi_{n}\right\|_{H_{0}^{\alpha / 2}}^{2}-\left\|\phi_{n}\right\|_{p}^{p}+o(1) \\
& =\left\langle I^{\prime}\left(u_{0}\right), u_{0}\right\rangle+\left\|\phi_{n}\right\|_{H_{0}^{\alpha / 2}}^{2}-\left\|\phi_{n}\right\|_{p}^{p}+o(1) \\
& =\left\|\phi_{n}\right\|_{H_{0}^{\alpha / 2}}^{2}-\left\|\phi_{n}\right\|_{p}^{p}+o(1) .
\end{aligned}
$$

Now we want to prove that $\left\{\phi_{n}\right\}$ has a subsequence strongly converging to 0 in $H_{0}^{\alpha / 2}(\Omega)$. Suppose, on the contrary, that there are $C, k>0$ such that $\left\|\phi_{n}\right\|_{H_{0}^{\alpha / 2}} \geq C$ for all $n \geq k$. Using (2-3) in (3-24), we get $\left\|\phi_{n}\right\|_{p}^{p-2} \geq S(\alpha, N)+o(1)$ and hence

$$
\left\|\phi_{n}\right\|_{p}^{p} \geq S(\alpha, N)^{N / \alpha}+o(1) .
$$

From (3-23) and (3-25), we have

$$
\begin{aligned}
\frac{\alpha}{2 N} S(\alpha, N)^{N / \alpha} & \leq \frac{\alpha}{2 N}\left\|\phi_{n}\right\|_{p}^{p}+o(1)=\frac{1}{2}\left\|\phi_{n}\right\|_{H_{0}^{\alpha / 2}}^{2}-\frac{1}{p}\left\|\phi_{n}\right\|_{p}^{p}+o(1) \\
& <\frac{\alpha}{2 N} S(\alpha, N)^{N / \alpha},
\end{aligned}
$$

which is a contradiction.

It is known (see, for instance, [Chen et al. 2006]) that the minimizers for the Sobolev inequality (2-4) are given by the two-parameter family of functions

$$
u_{\varepsilon, x_{0}}(x)=\frac{\varepsilon^{(N-\alpha) / 2}}{\left(\left|x-x_{0}\right|^{2}+\varepsilon^{2}\right)^{(N-\alpha) / 2}},
$$

where $x_{0} \in \mathbb{R}^{N}, \varepsilon>0$. In what follows we will use the notation

$$
A=\left\|u_{\varepsilon, x_{0}}\right\|_{p}, \quad B=\left\|(-\Delta)_{\mathscr{F}}^{\alpha / 4} u_{\varepsilon, x_{0}}\right\|_{2}=\left(\int_{\mathbb{R}^{N}}|\xi|^{\alpha}\left|\hat{u}_{\varepsilon, x_{0}}(\xi)\right|^{2} d \xi\right)^{1 / 2} .
$$

Note that the last quantity defines a norm in the homogeneous fractional Sobolev space $\dot{H}^{\alpha / 2}\left(\mathbb{R}^{N}\right)$. Both numbers $A$ and $B$ are clearly independent of $\varepsilon$ and $x_{0}$, and moreover $B^{2}=S(\alpha, N) A^{2}$.

Without loss of generality we may assume that $0 \in \Omega$. We define a cut-off function $\theta \in \mathscr{C}^{\infty}\left(\mathbb{R}^{N}\right)$ by $\theta(x)=\theta_{0}(|x| / \rho)$ with $\rho>0$, where $\theta_{0} \in \mathscr{C}^{\infty}(\mathbb{R})$ is a nonincreasing function satisfying

$$
\theta_{0}(s)=1 \quad \text { if } s \leq \frac{1}{2}, \quad \theta_{0}(s)=0 \quad \text { if } s \geq 1 .
$$

We now recall that, if $u_{0}$ is the solution constructed in the previous subsection, we can find a set $\Sigma \subset \Omega$ of positive Lebesgue measure such that $u_{0} \geq v>0$ a.e. 
in $\Sigma$ (replace $u_{0}$ with $-u_{0}$ and $f$ with $-f$ if necessary). For $x_{0} \in \Sigma$, we set $\tilde{u}_{\varepsilon, x_{0}}=\theta u_{\varepsilon, x_{0}} \in H_{0}^{\alpha / 2}(\Omega)$.

Proposition 3.8. In the above notation, for a.e. $x_{0} \in \Sigma$ there exists $\varepsilon^{*}=\varepsilon^{*}\left(x_{0}\right)>0$ sufficiently small such that

$$
\sup _{t \geq 0} I\left(u_{0}+t \tilde{u}_{\varepsilon, x_{0}}\right)<c^{*} \text { for all } 0<\varepsilon<\varepsilon^{*} .
$$

We observe that when one evaluates the functional in (3-28), one needs to evaluate $\left\|\tilde{u}_{\varepsilon, x_{0}}\right\|_{H_{0}^{\alpha / 2}}$; i.e., one needs to evaluate the fractional Laplacian of a product of functions. This requires the use of a different, but equivalent, norm which does not involve directly the fractional Laplacian. It uses the so-called $\alpha$-harmonic extension of [Caffarelli and Silvestre 2007] for $(-\Delta)_{\mathscr{F}}^{\alpha / 4}$, adapted to the bounded domain setting in [Brändle et al. 2013; Cabré and Tan 2010; Stinga and Torrea 2010].

Consider the semi-infinite cylinder $\mathscr{C}_{\Omega}=\{(x, y): x \in \Omega, y>0\} \subset \mathbb{R}_{+}^{N+1}$ and its lateral boundary $\partial_{L} \mathfrak{C}_{\Omega}=\partial \Omega \times(0, \infty)$. For a function $u \in H_{0}^{\alpha / 2}(\Omega)$, we denote its $\alpha$-harmonic extension to $\mathscr{C}_{\Omega}$ by $w=\mathrm{E}_{\alpha}(u)$, defined as the solution to the problem

$$
\begin{cases}\operatorname{div}\left(y^{1-\alpha} \nabla w\right)=0 & \text { in } \mathscr{C}_{\Omega}, \\ w=0 & \text { on } \partial_{L} \mathscr{b}_{\Omega}, \\ w=u & \text { on } \Omega \times\{y=0\} .\end{cases}
$$

Then the equation

$$
-\kappa_{\alpha} \lim _{y \searrow 0} \frac{\partial w}{\partial y}=(-\Delta)^{\alpha / 2} u
$$

holds, with $\kappa_{\alpha}$ a positive constant. Let $X_{0}^{\alpha}\left(\mathscr{C}_{\Omega}\right)$ be the completion of $\mathscr{C}_{0}^{\infty}(\Omega \times[0, \infty))$ under the norm

$$
\|\phi\|_{X_{0}^{\alpha}}=\left(\kappa_{\alpha} \int_{\mathscr{C}_{\Omega}} y^{1-\alpha}|\nabla \phi(x, y)|^{2} d x d y\right)^{1 / 2} .
$$

In the case $\Omega=\mathbb{R}^{N}$ the corresponding space for functions in the upper halfspace $\mathbb{R}_{+}^{N+1}$ is $X^{\alpha}\left(\mathbb{R}_{+}^{N+1}\right)$, which can be defined in the same way with the integral extended to $\mathbb{R}_{+}^{N+1}$. The extension operator can be characterized also as a minimization of the $X_{0}^{\alpha}$-norm (equivalently, the $X^{\alpha}$ norm) for all the functions with common trace at $y=0$. Note that the extension operator is an isometry from $H_{0}^{\alpha / 2}(\Omega)$ to $X_{0}^{\alpha}\left(\mathfrak{C}_{\Omega}\right)$ and from $H^{\alpha / 2}\left(\mathbb{R}^{N}\right)$ to $X^{\alpha}\left(\mathbb{R}_{+}^{N+1}\right)$; that is,

$$
\begin{array}{cc}
\left\|\mathrm{E}_{\alpha}(\psi)\right\|_{X_{0}^{\alpha}}=\|\psi\|_{H_{0}^{\alpha / 2}} & \text { for all } \psi \in H_{0}^{\alpha / 2}(\Omega), \\
\left\|\mathrm{E}_{\alpha}(\psi)\right\|_{X^{\alpha}}=\|\psi\|_{\dot{H}^{\alpha / 2}} & \text { for all } \psi \in \dot{H}^{\alpha / 2}\left(\mathbb{R}^{N}\right) .
\end{array}
$$

This means that

$$
\|w\|_{X_{0}^{\alpha}} \geq\|w(\cdot, 0)\|_{H_{0}^{\alpha / 2}} \text { for all } w \in X_{0}^{\alpha}\left(\mathscr{C}_{\Omega}\right)
$$


and

$$
\|w\|_{X^{\alpha}} \geq\|w(\cdot, 0)\|_{H^{\alpha / 2}} \text { for all } w \in X^{\alpha}\left(\mathbb{R}^{N}\right) .
$$

See [Brändle et al. 2013] for the details.

We define the family $w_{\varepsilon, x_{0}}=E_{\alpha}\left(u_{\varepsilon, x_{0}}\right)$, with $u_{\varepsilon, x_{0}}$ given in (3-26). We want to find a family of modified minimizers in the extended space, by using a cutoff function in $\mathscr{C}_{\Omega}$. To do that we take

$$
\phi(x, y)=\theta_{0}\left(\frac{\left(\left|x-x_{0}\right|^{2}+y^{2}\right)^{1 / 2}}{\rho}\right),
$$

where $\theta_{0}$ is defined above. With this notation we define $\tilde{w}_{\varepsilon, x_{0}}=\phi w_{\varepsilon, x_{0}} \in X_{0}^{\alpha}\left(\mathscr{C}_{\Omega}\right)$ and $\tilde{w}_{\varepsilon, x_{0}}(\cdot, 0)=\tilde{u}_{\varepsilon, x_{0}}(\cdot)$.

In [Barrios et al. 2012, Lemma 3.8] the following estimates for $\tilde{w}_{\varepsilon, x_{0}}$ are proved:

$$
\left\|\tilde{w}_{\varepsilon, x_{0}}\right\|_{X_{0}^{\alpha}}^{2}=\left\|w_{\varepsilon, x_{0}}\right\|_{X^{\alpha}}^{2}+O\left(\varepsilon^{N-\alpha}\right) .
$$

In view of (3-30), (3-32) and (3-34), we have

$$
\left\|\tilde{u}_{\varepsilon, x_{0}}\right\|_{H_{0}^{\alpha / 2}}^{2} \leq B^{2}+O\left(\varepsilon^{N-\alpha}\right) .
$$

Moreover,

$$
\left\|\tilde{u}_{\varepsilon, x_{0}}\right\|_{p}^{p} \geq A^{p}+O\left(\varepsilon^{N}\right) .
$$

We now state a result that will be useful in the proof of Proposition 3.8. Its proof follows the same arguments as in [Brézis and Nirenberg 1989], with obvious changes for our setting, so we omit the details.

Lemma 3.9. Assume that $a, b>0$ and that $u_{0}, \tilde{u}_{\varepsilon, x_{0}}$ are defined as above. For $t \in[a, b]$, we have

$$
\begin{aligned}
\left\|u_{0}+t \tilde{u}_{\varepsilon, x_{0}}\right\|_{p}^{p}=\left\|u_{0}\right\|_{p}^{p}+t^{p}\left\|\tilde{u}_{\varepsilon, x_{0}}\right\|_{p}^{p}+p t \int_{\Omega}\left|u_{0}\right|^{p-2} u_{0} \tilde{u}_{\varepsilon, x_{0}} \\
\\
\quad+p t^{p-1} \int_{\Omega}\left|\tilde{u}_{\varepsilon, x_{0}}\right|^{p-2} \tilde{u}_{\varepsilon, x_{0}} u_{0}+o\left(\varepsilon^{(N-\alpha) / 2}\right) .
\end{aligned}
$$

Proof of Proposition 3.8. On the one hand, since $\left.I\left(u_{0}+t \tilde{u}_{\varepsilon, x_{0}}\right)\right|_{t=0}=c_{0}<c^{*}$, by a continuity argument we can find $t_{0}, \varepsilon_{0}>0$ both small enough such that

$$
I\left(u_{0}+t \tilde{u}_{\varepsilon, x_{0}}\right)<c^{*} \quad \text { for all } t \in\left(0, t_{0}\right) \text { and all } \varepsilon \in\left(0, \varepsilon_{0}\right) .
$$

On the other hand, by Lemma 3.9, together with (3-36) and the fact that $A$ and $B$ are independent of $\varepsilon$, we have

$$
I\left(u_{0}+t \tilde{u}_{\varepsilon, x_{0}}\right) \rightarrow-\infty \text { as } t \rightarrow \infty \text { for all } \varepsilon>0 .
$$


Hence there exists $t_{1}>0$ large enough that

$$
I\left(u_{0}+t \tilde{u}_{\varepsilon, x_{0}}\right)<c_{0}<c^{*} \text { for all } t \geq t_{1} \text { and all } \varepsilon \in\left(0, \varepsilon_{0}\right) .
$$

Thus, we just need to prove that there exists $\varepsilon^{*} \in\left(0, \varepsilon_{0}\right)$ such that

$$
\sup _{t_{0} \leq t \leq t_{1}} I\left(u_{0}+t \tilde{u}_{\varepsilon, x_{0}}\right)<c^{*}
$$

for every $0<\varepsilon<\varepsilon^{*}$.

Take $t \in\left[t_{0}, t_{1}\right]$. Clearly, we have

$$
\begin{array}{r}
I\left(u_{0}+t \tilde{u}_{\varepsilon, x_{0}}\right)=\frac{1}{2}\left\|u_{0}\right\|_{H_{0}^{\alpha / 2}}^{2}+t \int_{\Omega}(-\Delta)^{\alpha / 4} u_{0}(-\Delta)^{\alpha / 4} \tilde{u}_{\varepsilon, x_{0}}+\frac{t^{2}}{2}\left\|\tilde{u}_{\varepsilon, x_{0}}\right\|_{H_{0}^{\alpha / 2}}^{2} \\
-\frac{1}{p}\left\|u_{0}+t \tilde{u}_{\varepsilon, x_{0}}\right\|_{p}^{p}-\int_{\Omega} f u_{0}-t \int_{\Omega} f \tilde{u}_{\varepsilon, x_{0}} .
\end{array}
$$

Since $S(\alpha, N)$ is attained for the function $u_{\varepsilon, x_{0}}$, substituting (3-35), (3-36) and (3-37) in (3-38) we have

$$
\begin{gathered}
I\left(u_{0}+t \tilde{u}_{\varepsilon, x_{0}}\right) \leq \frac{1}{2}\left\|u_{0}\right\|_{H_{0}^{\alpha / 2}}^{2}+t \int_{\Omega}(-\Delta)^{\alpha / 4} u_{0}(-\Delta)^{\alpha / 4} \tilde{u}_{\varepsilon, x_{0}}+\frac{t^{2}}{2} B^{2} \\
-\frac{1}{p}\left\|u_{0}\right\|_{p}^{p}-\frac{t^{p}}{p} A^{p}-t \int_{\Omega}\left|u_{0}\right|^{p-2} u_{0} \tilde{u}_{\varepsilon, x_{0}}-t^{p-1} \int_{\Omega}\left|\tilde{u}_{\varepsilon, x_{0}}\right|^{p-1} u_{0} \\
\quad-\int_{\Omega} f u_{0}-t \int_{\Omega} f \tilde{u}_{\varepsilon, x_{0}}+o\left(\varepsilon^{(N-\alpha) / 2}\right) .
\end{gathered}
$$

On the other hand, since $u_{0}$ is solution of $(\mathrm{P})$, we get

$$
I\left(u_{0}+t \tilde{u}_{\varepsilon, x_{0}}\right) \leq I\left(u_{0}\right)+\frac{t^{2}}{2} B^{2}-t^{p-1} \int_{\Omega}\left|\tilde{u}_{\varepsilon, x_{0}}\right|^{p-1} u_{0}-\frac{t^{p}}{p} A^{p}+o\left(\varepsilon^{(N-\alpha) / 2}\right) .
$$

Extending $u_{0}$ by zero outside $\Omega$, we get

$$
\begin{aligned}
\int_{\Omega}\left|\tilde{u}_{\varepsilon, x_{0}}\right|^{p-1} u_{0} & =\int_{\mathbb{R}^{N}} u_{0}(x) \theta^{p-1}(x) \frac{\varepsilon^{(N+\alpha) / 2}}{\left(\left|x-x_{0}\right|^{2}+\varepsilon^{2}\right)^{(N+\alpha) / 2}} \\
& =\varepsilon^{(N-\alpha) / 2} \int_{\mathbb{R}^{N}} u_{0}(x) \theta^{p-1}(x) \frac{1}{\varepsilon^{N}} \eta\left(\frac{x-x_{0}}{\varepsilon}\right),
\end{aligned}
$$

with $\eta(x)=\left(|x|^{2}+1\right)^{-(N+\alpha) / 2}$. Thus, there exists a constant $v>0$ such that

$$
\int_{\mathbb{R}^{N}} u_{0}(x) \theta^{p-1}(x) \frac{1}{\varepsilon^{N}} \eta\left(\frac{x-x_{0}}{\varepsilon}\right) \geq K v
$$

for every $\varepsilon>0$ sufficiently small, $x_{0} \in \Sigma$ and $K=\int_{\mathbb{R}^{N}} \eta(x)<\infty$. Therefore

$$
\int_{\Omega}\left|\tilde{u}_{\varepsilon, x_{0}}\right|^{p-1} u_{0}=\varepsilon^{(N-\alpha) / 2} K v+o\left(\varepsilon^{(N-\alpha) / 2}\right) .
$$

Substituting (3-40) in (3-39), we have

$$
I\left(u_{0}+t \tilde{u}_{\varepsilon, x_{0}}\right) \leq c_{0}+\frac{t^{2}}{2} B^{2}-t^{p-1} \varepsilon^{(N-\alpha) / 2} K v-\frac{t^{p}}{p} A^{p}+o\left(\varepsilon^{(N-\alpha) / 2}\right) .
$$


Let us now define the function

$$
g(s)=\frac{s^{2}}{2} B^{2}-s^{p-1} \varepsilon^{(N-\alpha) / 2} K v-\frac{s^{p}}{p} A^{p} \quad \text { for } s>0,
$$

and let $s_{\varepsilon}>0$ be the point of global maximum, i.e.,

$$
0=g^{\prime}\left(s_{\varepsilon}\right)=s_{\varepsilon} B^{2}-(p-1) s_{\varepsilon}^{p-2} \varepsilon^{(N-\alpha) / 2} K v-s_{\varepsilon}^{p-1} A^{p} .
$$

We denote $S_{0}=\left(B^{2} / A^{p}\right)^{1 /(p-2)}$. Note that $0<s_{\varepsilon}<S_{0}$ and $s_{\varepsilon} \rightarrow S_{0}$ as $\varepsilon \searrow 0$. Let $\delta_{\varepsilon}>0$ be such that $s_{\varepsilon}=S_{0}\left(1-\delta_{\varepsilon}\right)$. Since $B^{2} / A^{p}=S_{0}^{p-2}$, by (3-42) we have $\left(\frac{B^{2(p-1)}}{A^{p}}\right)^{1 /(p-2)}\left(1-\delta_{\varepsilon}-\left(1-\delta_{\varepsilon}\right)^{p-1}\right)-(p-1) S_{0}^{p-2}\left(1-\delta_{\varepsilon}\right)^{p-2} \varepsilon^{(N-\alpha) / 2} K v=0$, which implies

$$
(p-2)\left(\frac{B^{2(p-1)}}{A^{p}}\right)^{1 /(p-2)} \delta_{\varepsilon}=(p-1) S_{0}^{p-2} \varepsilon^{(N-\alpha) / 2} K v+o\left(\varepsilon^{(N-\alpha) / 2}\right) .
$$

By (3-41) with $t=s_{\varepsilon}$ and (3-43), we have

$$
\begin{aligned}
I\left(u_{0}+s_{\varepsilon} \tilde{u}_{\varepsilon, x_{0}}\right) & \leq c_{0}+\frac{s_{\varepsilon}^{2}}{2} B^{2}-s_{\varepsilon}^{p-1} \varepsilon^{(N-\alpha) / 2} K v-\frac{s_{\varepsilon}^{p}}{p} A^{p}+o\left(\varepsilon^{(N-\alpha) / 2}\right) \\
& =c_{0}+\frac{S_{0}^{2}}{2} B^{2}-S_{0}^{p-1} \varepsilon^{(N-\alpha) / 2} K v-\frac{S_{0}^{p}}{p} A^{p}+o\left(\varepsilon^{(N-\alpha) / 2}\right) \\
& =c_{0}+\frac{\alpha}{2 N} S(\alpha, N)^{N / \alpha}-S_{0}^{p-1} \varepsilon^{(N-\alpha) / 2} K v+o\left(\varepsilon^{(N-\alpha) / 2}\right) \\
& =c^{*}-S_{0}^{p-1} \varepsilon^{(N-\alpha) / 2} K v+o\left(\varepsilon^{(N-\alpha) / 2}\right) .
\end{aligned}
$$

Taking $\varepsilon$ sufficiently small, this finishes the proof.

Lemma 3.10. Assume $f \not \equiv 0$ satisfies (2-1). Then the functional I possesses a critical point different from $u_{0}$. In particular, $(\mathrm{P})$ has a second solution. Moreover, if $f \geq 0$ a.e. in $\Omega$ then this solution is nonnegative a.e. in $\Omega$.

Proof. Set $\eta_{\varepsilon, M}=u_{0}+M \tilde{u}_{\varepsilon, x_{0}}$, with $0<\varepsilon<\varepsilon^{*}$ and $x_{0} \in \Sigma$ so that (3-28) holds. Assume that $M>0$ is large enough such that $I\left(\eta_{\varepsilon, M}\right)<c_{0}$.

Now we set

$$
\Gamma=\left\{\gamma:[0,1] \rightarrow H_{0}^{\alpha / 2}(\Omega) \text { such that } \gamma(0)=u_{0}, \gamma(1)=\eta_{\varepsilon, M}\right\} .
$$

By Proposition 3.8 we have

$$
c_{0}<c_{1}=\inf _{\gamma \in \Gamma} \max _{t \in[0,1]} I(\gamma(t))<c^{*} .
$$

Thus, using the mountain pass theorem, we obtain a PS sequence of level $c_{1}$, and as a consequence of Lemma 3.7 we can find a critical point $u_{1}$ in $H_{0}^{\alpha / 2}(\Omega)$ with energy level $c_{1}>c_{0}$, i.e., $u_{1}$ is a solution of $(\mathrm{P})$ with $u_{1} \not \equiv u_{0}$. 
To prove that the solution is positive in the case that $f \geq 0$, we set

$$
\tilde{\mathcal{N}}:=\{u \in \mathcal{N}: u \text { satisfies }(3-6)\}
$$

and $c_{2}=\inf _{\tilde{\mathcal{N}}} I$. Is easy to see that, taking a larger $M$ if necessary, we can assume

$$
c_{0}<c_{2} \leq c_{1}<c^{*} \text {. }
$$

Now, using Ekeland's variational principle and following the steps of the proof of Proposition 3.6, we can obtain a PS sequence of level $c_{2}$. Again, Lemma 3.7 implies the existence of a solution $u_{2} \in \mathcal{N}$ such that $I\left(u_{2}\right)=c_{2}$. Put $\tau=\tau\left(\left|u_{2}\right|\right)>0$. Then $\tau\left|u_{2}\right| \in \tilde{\mathcal{N}}$. Finally, by Corollary 3.2,

$$
\inf _{\tilde{\mathcal{N}}} I=I\left(u_{2}\right)=\max _{t \geq t_{M}} I\left(t u_{2}\right) \geq I\left(\tau u_{2}\right) \geq I\left(\tau\left|u_{2}\right|\right),
$$

which finishes the proof.

Remark. Note that $u_{2}$ could coincide with $u_{1}$.

\section{Proof of Theorem 2.2}

When $f$ satisfies condition (2-2) instead of (2-1), we use an approximation argument. Proof of Theorem 2.2. Consider a sequence of numbers $\left\{\varepsilon_{k}\right\}_{k \in \mathbb{N}} \subset(0,1)$ such that $\varepsilon_{k} \searrow 0$ as $k \rightarrow \infty$, and define $f_{k}=\left(1-\varepsilon_{k}\right) f$. Clearly $f_{k}$ satisfies condition (2-1) for every $k \in \mathbb{N}$. We define $I_{k}$ and $\mathcal{N}_{k}$ in a natural way:

$$
\begin{aligned}
I_{k}(u) & =\frac{1}{2} \int_{\Omega}\left|(-\Delta)^{\alpha / 4} u\right|^{2}-\frac{1}{p} \int_{\Omega}|u|^{p}-\int_{\Omega} f_{k} u, \\
\mathcal{N}_{k} & =\left\{u \in H_{0}^{\alpha / 2}(\Omega): u \neq \equiv,\left\langle I_{k}^{\prime}(u), u\right\rangle=0\right\} .
\end{aligned}
$$

Let $u_{k} \in \mathcal{N}_{k}$ be the local minimum found via Theorem 2.1, namely,

In particular, we have

$$
I_{k}\left(u_{k}\right)=\inf _{\mathcal{N}_{k}} I_{k}:=c_{k}
$$

$$
\left\langle I_{k}^{\prime}\left(u_{k}\right), z\right\rangle=0 \quad \text { for all } z \in H_{0}^{\alpha / 2}(\Omega),
$$

and moreover

$$
\left\|u_{k}\right\|_{H_{0}^{\alpha / 2}}^{2}-\left\|u_{k}\right\|_{p}^{p}-\int_{\Omega} f_{k} u_{k}=0,
$$

which, by (2-3) and (2-6), implies that $\left\|u_{k}\right\|_{H_{0}^{\alpha / 2}}^{2}<C$ for any $k \in \mathbb{N}$ and some constant $C>0$ independent of $k$. Take $u \in \mathcal{N}$ satisfying (3-5). Then

$$
\int_{\Omega} f_{k} u>0 \text { for all } k \in \mathbb{N} \text {. }
$$


Applying Lemma 3.1 with $f=f_{k}$ and $\mathcal{N}=\mathcal{N}_{k}$, we find the values $0<\sigma_{k}<t_{M_{k}}<\tau_{k}$ such that $\sigma_{k} u, \tau_{k} u \in \mathcal{N}_{k}$. Since $u$ satisfies the inequality (3-5), we have $\tau_{k}>1$. Thus, by Corollary 3.2 we have $I_{k}\left(\sigma_{k} u\right) \leq I_{k}(u)$, which leads to

$$
c_{k} \leq I_{k}\left(\sigma_{k} u\right) \leq I_{k}(u) \leq I(u)+\varepsilon_{k}\|f\|_{H^{-\alpha / 2}}\|u\|_{H_{0}^{\alpha / 2}} \leq I(u)+C \varepsilon_{k} .
$$

In particular, $c_{k} \leq c_{0}+C \varepsilon_{k}$. Finally, reasoning as in (3-13) with $f=f_{k}$, we obtain

$$
-\frac{(N+\alpha)^{2}}{8 N \alpha}\|f\|_{H^{-\alpha / 2}}^{2}<-\frac{(N+\alpha)^{2}}{8 N \alpha}\left\|f_{k}\right\|_{H^{-\alpha / 2}}^{2} \leq c_{k} \leq c_{0}+C \varepsilon_{k} .
$$

After passing to a subsequence, we can assume that $c_{k}$ converges to some value $c^{\prime}$ such that

$$
-\frac{(N+\alpha)^{2}}{8 N \alpha}\|f\|_{H^{-\alpha / 2}}^{2} \leq c^{\prime} \leq c_{0} .
$$

Moreover, since $\left\|u_{k}\right\|_{H_{0}^{\alpha / 2}}^{2}$ is uniformly bounded, again for a subsequence if necessary, we have $u_{k} \rightarrow u^{*}$ weakly in $H_{0}^{\alpha / 2}(\Omega)$. Then by (4-1) we have

$$
\left\langle I^{\prime}\left(u^{*}\right), z\right\rangle=0 \text { for all } z \in H_{0}^{\alpha / 2}(\Omega),
$$

and $I\left(u^{*}\right) \leq c_{0}$. This implies $u^{*} \in \mathcal{N}$ and $I\left(u^{*}\right)=c_{0}$, which finishes the proof. The positivity of the solution when the datum $f$ is taken nonnegative follows from the same argument as in the proof of Theorem 2.1.

We finally remark that the solution constructed in this way is not necessarily a minimum of the functional. Therefore we cannot prove the mountain pass geometry in order to find a second solution.

\section{References}

[Ambrosetti and Rabinowitz 1973] A. Ambrosetti and P. H. Rabinowitz, "Dual variational methods in critical point theory and applications", J. Funct. Anal. 14 (1973), 349-381. MR 51 \#6412 Zbl 0273.49063

[Ambrosetti et al. 1994] A. Ambrosetti, H. Brézis, and G. Cerami, "Combined effects of concave and convex nonlinearities in some elliptic problems", J. Funct. Anal. 122:2 (1994), 519-543. MR 95g:35059 Zbl 0805.35028

[Barrios et al. 2012] B. Barrios, E. Colorado, A. de Pablo, and U. Sánchez, "On some critical problems for the fractional Laplacian operator", J. Differential Equations 252:11 (2012), 6133-6162. MR 2911424 Zbl 1245.35034

[Barrios et al. 2014] B. Barrios, E. Colorado, R. Servadei, and F. Soria, "A critical fractional equation with concave-convex power nonlinearities", Ann. Inst. H. Poincaré Anal. Non Linéaire (2014). To appear.

[Brändle et al. 2013] C. Brändle, E. Colorado, A. de Pablo, and U. Sánchez, "A concave-convex elliptic problem involving the fractional Laplacian", Proc. Roy. Soc. Edinburgh Sect. A 143:1 (2013), 39-71. MR 3023003 Zbl 06238478

[Brézis and Lieb 1983] H. Brézis and E. Lieb, "A relation between pointwise convergence of functions and convergence of functionals", Proc. Amer. Math. Soc. 88:3 (1983), 486-490. MR 84e:28003 Zbl 0526.46037 
[Brézis and Nirenberg 1983] H. Brézis and L. Nirenberg, "Positive solutions of nonlinear elliptic equations involving critical Sobolev exponents", Comm. Pure Appl. Math. 36:4 (1983), 437-477. MR 84h:35059 Zbl 0541.35029

[Brézis and Nirenberg 1989] H. Brézis and L. Nirenberg, "A minimization problem with critical exponent and nonzero data", pp. 129-140 in Symmetry in nature (Pisa, 1989), edited by G. Bernardini, Scuola Normale Superiore, Pisa, 1989. Reprinted by Springer, Berlin, 2007. Zbl 0763.46023

[Cabré and Tan 2010] X. Cabré and J. Tan, "Positive solutions of nonlinear problems involving the square root of the Laplacian", Adv. Math. 224:5 (2010), 2052-2093. MR 2011c:35106 Zbl 1198.35286

[Caffarelli and Silvestre 2007] L. Caffarelli and L. Silvestre, "An extension problem related to the fractional Laplacian”, Comm. Partial Differential Equations 32:7-9 (2007), 1245-1260. MR 2009k: 35096 Zbl 1143.26002

[Castro and Zuluaga 1993] R. Castro and M. Zuluaga, "Existence results for a class of nonhomogeneous elliptic equations with critical Sobolev exponent", Note Mat. 13:2 (1993), 269-276. MR 96b:35060 Zbl 0831.35060

[Chen et al. 2006] W. Chen, C. Li, and B. Ou, "Classification of solutions for an integral equation", Comm. Pure Appl. Math. 59:3 (2006), 330-343. MR 2006m:45007a Zbl 1093.45001

[Ekeland 1974] I. Ekeland, "On the variational principle”, J. Math. Anal. Appl. 47 (1974), 324-353. MR 49 \#11344 Zbl 0286.49015

[Hardy and Littlewood 1928] G. H. Hardy and J. E. Littlewood, "Some properties of fractional integrals, I”, Math. Z. 27:1 (1928), 565-606. MR 1544927 JFM 54.0275.05

[Lieb 1983] E. H. Lieb, "Sharp constants in the Hardy-Littlewood-Sobolev and related inequalities", Ann. of Math. (2) 118:2 (1983), 349-374. MR 86i:42010 Zbl 0527.42011

[Lions 1985] P.-L. Lions, "The concentration-compactness principle in the calculus of variations: the limit case, II”, Rev. Mat. Iberoamericana 1:2 (1985), 45-121. MR 87j:49012 Zbl 0704.49006

[de Pablo et al. 2012] A. de Pablo, F. Quirós, A. Rodríguez, and J. L. Vázquez, "A general fractional porous medium equation", Comm. Pure Appl. Math. 65:9 (2012), 1242-1284. MR 2954615 Zbl 1248.35220

[Pohožaev 1970] S. I. Pohožaev, “О собственных функциях квазилинейных эллиптических задач", Mat. Sb. (N.S.) 124:2 (1970), 192-212. Translated as "On the eigenfunctions of quasilinear elliptic problems" in Math. USSR Sb. 11:2 (1970), 171-188. MR 42 \#8081 Zbl 0217.13203

[Rey 1992] O. Rey, "Concentration of solutions to elliptic equations with critical nonlinearity", Ann. Inst. H. Poincaré Anal. Non Linéaire 9:2 (1992), 201-218. MR 93e:35040 Zbl 0761.35034

[Servadei and Valdinoci $\geq 2014$ ] R. Servadei and E. Valdinoci, "The Brézis-Nirenberg result for the fractional Laplacian", Trans. Amer. Math. Soc.. To appear.

[Sobolev 1938] S. L. Sobolev, “Об одной теореме функционального анализа”, Mat. Sb. (N.S.) 46:3 (1938), 471-497. Translated as "On a theorem of functional analysis" in Eleven Papers on Analysis, Amer. Math. Soc. Transl. (2) 34 (1963), 39-68. JFM 64.1100.02

[Stinga and Torrea 2010] P. R. Stinga and J. L. Torrea, "Extension problem and Harnack's inequality for some fractional operators", Comm. Partial Differential Equations 35:11 (2010), 2092-2122. MR 2012c:35456 Zbl 1209.26013

[Tan 2011] J. Tan, "The Brézis-Nirenberg type problem involving the square root of the Laplacian", Calc. Var. Partial Differential Equations 42:1-2 (2011), 21-41. MR 2012e:35079 Zbl 1248.35078

[Tarantello 1992] G. Tarantello, "On nonhomogeneous elliptic equations involving critical Sobolev exponent”, Ann. Inst. H. Poincaré Anal. Non Linéaire 9:3 (1992), 281-304. MR 93i:35043 Zbl 0785.35046 
Received April 11, 2013. Revised July 8, 2013.

EDUARDO COLORADO

DEPARTAMENTO DE MATEMÁTICAS

UNIVERSIDAD CARLOS III DE MADRID

AVENIDA UNIVERSIDAD 30

28911 LEGANÉS

SPAIN

ecolorad@math.uc3m.es

and

Instituto de Ciencias Matemáticas, ICMAT (CSIC-UAM-UC3M-UCM)

C/ NiCOLÁS CABRERA 15

28049 MADRID

SPAIN.

eduardo.colorado@icmat.es

ARturo de Pablo

DEPARTAMENTO DE MATEMÁTICAS

UNIVERSIDAD CARLOS III DE MADRID

AVENIDA UNIVERSIDAD 30

28911 LEGANÉS

SPAIN

arturop@math.uc3m.es

URKO SÁNCHEZ

UNIVERSIDAD CARLOS III DE MADRID

28911 LEGANÉS

SPAIN

urko.sanchez@gmail.com 


\title{
PACIFIC JOURNAL OF MATHEMATICS
}

\author{
msp.org/pjm
}

Founded in 1951 by E. F. Beckenbach (1906-1982) and F. Wolf (1904-1989)

\section{EDITORS}

Don Blasius (Managing Editor)

Department of Mathematics

University of California

Los Angeles, CA 90095-1555

blasius@math.ucla.edu

\author{
Paul Balmer \\ Department of Mathematics \\ University of California \\ Los Angeles, CA 90095-1555 \\ balmer@math.ucla.edu \\ Robert Finn \\ Department of Mathematics \\ Stanford University \\ Stanford, CA 94305-2125 \\ finn@math.stanford.edu \\ Sorin Popa \\ Department of Mathematics \\ University of California \\ Los Angeles, CA 90095-1555 \\ popa@math.ucla.edu
}

\author{
Vyjayanthi Chari \\ Department of Mathematics \\ University of California \\ Riverside, CA 92521-0135 \\ chari@math.ucr.edu \\ Kefeng Liu \\ Department of Mathematics \\ University of California \\ Los Angeles, CA 90095-1555 \\ liu@math.ucla.edu \\ Jie Qing \\ Department of Mathematics \\ University of California \\ Santa Cruz, CA 95064 \\ qing@ cats.ucsc.edu
}

\section{PRODUCTION}

Silvio Levy, Scientific Editor, production@msp.org

\section{SUPPORTING INSTITUTIONS}

ACADEMIA SINICA, TAIPEI

CALIFORNIA INST. OF TECHNOLOGY

INST. DE MATEMÁTICA PURA E APLICADA

KEIO UNIVERSITY

MATH. SCIENCES RESEARCH INSTITUTE

NEW MEXICO STATE UNIV.

OREGON STATE UNIV.

\author{
STANFORD UNIVERSITY \\ UNIV. OF BRITISH COLUMBIA \\ UNIV. OF CALIFORNIA, BERKELEY \\ UNIV. OF CALIFORNIA, DAVIS \\ UNIV. OF CALIFORNIA, LOS ANGELES \\ UNIV. OF CALIFORNIA, RIVERSIDE \\ UNIV. OF CALIFORNIA, SAN DIEGO \\ UNIV. OF CALIF., SANTA BARBARA
}

\author{
Daryl Cooper \\ Department of Mathematics \\ University of California \\ Santa Barbara, CA 93106-3080 \\ cooper@math.ucsb.edu \\ Jiang-Hua Lu \\ Department of Mathematics \\ The University of Hong Kong \\ Pokfulam Rd., Hong Kong \\ jhlu@maths.hku.hk \\ Paul Yang \\ Department of Mathematics \\ Princeton University \\ Princeton NJ 08544-1000 \\ yang@math.princeton.edu
}

These supporting institutions contribute to the cost of publication of this Journal, but they are not owners or publishers and have no responsibility for its contents or policies.

See inside back cover or msp.org/pjm for submission instructions.

The subscription price for 2014 is US $\$ 410 /$ year for the electronic version, and \$535/year for print and electronic.

Subscriptions, requests for back issues and changes of subscribers address should be sent to Pacific Journal of Mathematics, P.O. Box 4163, Berkeley, CA 94704-0163, U.S.A. The Pacific Journal of Mathematics is indexed by Mathematical Reviews, Zentralblatt MATH, PASCAL CNRS Index, Referativnyi Zhurnal, Current Mathematical Publications and Web of Knowledge (Science Citation Index).

The Pacific Journal of Mathematics (ISSN 0030-8730) at the University of California, c/o Department of Mathematics, 798 Evans Hall \#3840, Berkeley, CA 94720-3840, is published twelve times a year. Periodical rate postage paid at Berkeley, CA 94704, and additional mailing offices. POSTMASTER: send address changes to Pacific Journal of Mathematics, P.O. Box 4163, Berkeley, CA 94704-0163.

PJM peer review and production are managed by EditFLOW ${ }^{\circledR}$ from Mathematical Sciences Publishers.

\section{PUBLISHED BY}

\section{mathematical sciences publishers \\ nonprofit scientific publishing}

http://msp.org/

(C) 2014 Mathematical Sciences Publishers 


\section{PACIFIC JOURNAL OF MATHEMATICS}

Volume $271 \quad$ No. $1 \quad$ September 2014

Proper holomorphic maps between bounded symmetric domains revisited 1

GAUTAM BHARALI and JAIKRISHNAN JANARDHANAN

An explicit Majorana representation of the group $3^{2}: 2$ of $3 C$-pure type 25

HSIAN-YANG CHEN and CHING HUNG LAM

Sofic groups: graph products and graphs of groups

LAUra Ciobanu, DereK F. Holt and SARAh ReES

Perturbations of a critical fractional equation

Eduardo Colorado, Arturo de Pablo and Urko SÁnchez

A density theorem in parametrized differential Galois theory

THOMAS DREYFUS

On the classification of complete area-stationary and stable surfaces in $\quad 143$ the subriemannian Sol manifold

MATteo Galli

Periodic orbits of Hamiltonian systems linear and hyperbolic at infinity

BAŞAK Z. GÜREL

Nonsplittability of the rational homology cobordism group of

3-manifolds

SE-Goo KIM and CHARLES LIVINGSTON

Biharmonic surfaces of constant mean curvature

ERIC LOUBEAU and CEZAR ONICIUC

Foliations of a smooth metric measure space by hypersurfaces with constant $f$-mean curvature

JUNCHEOL PYO

On the existence of large degree Galois representations for fields of small 243 discriminant

JEREMY ROUSE and FRANK THORNE 